\title{
P300 Detection Based on Feature Extraction in On-line Brain-Computer Interface
}

\author{
Nikolay Chumerin ${ }^{1, \star}$, Nikolay V. Manyakov ${ }^{1, *}$, Adrien Combaz ${ }^{1, *}$, \\ Johan A.K. Suykens ${ }^{2, \star \star}$, Refet Firat Yazicioglu ${ }^{3}$, Tom Torfs ${ }^{3}$, Patrick Merken ${ }^{3}$, \\ Herc P. Neves ${ }^{3}$, Chris Van Hoof ${ }^{3}$, and Marc M. Van Hulle ${ }^{3}$ \\ ${ }^{1}$ Laboratorium voor Neuro- en Psychofysiologie, K.U. Leuven, \\ Herestraat 49, bus 1021, 3000 Leuven, Belgium \\ 2 ESAT-SCD, K.U. Leuven, Kasteelpark Arenberg 10, 3001 Heverlee, Belgium \\ ${ }^{3}$ IMEC, Kapeldreef 75, 3001 Leuven, Belgium \\ \{Nikolay. Chumerin, NikolayV. Manyakov, Adrien.Combaz, \\ Marc.VanHulle\}@med.kuleuven. be, \\ Johan. Suykens@esat.kuleuven. be \\ \{Firat,TorfsT, Patrick.Merken, Chris.VanHoof , Herc\}@imec.be
}

\begin{abstract}
We propose a new EEG-based wireless brain computer interface (BCI) with which subjects can "mind-type" text on a computer screen. The application is based on detecting P300 event-related potentials in EEG signals recorded on the scalp of the subject. The BCI uses a simple classifier which relies on a linear feature extraction approach. The accuracy of the presented system is comparable to the state-of-the-art for on-line P300 detection, but with the additional benefit that its much simpler design supports a power-efficient on-chip implementation.
\end{abstract}

\section{Introduction}

Research on brain computer interfaces (BCIs) has witnessed a tremendous development in recent years (see, for example, the editorial in Nature 11), and is now widely considered as one of the most successful applications of the neurosciences. BCIs can significantly improve the quality of life of neurologically impaired patients with pathologies such as: amyotrophic lateral sclerosis, brain stroke, brain/spinal cord injury, cerebral palsy, muscular dystrophy, etc.

\footnotetext{
* NC is supported by the European Commission (STREP-2002-016276), NVM and AC are supported by the European Commission (IST-2004-027017), MMVH is supported by research grants received from the Excellence Financing program (EF 2005) and the CREA Financing program (CREA/07/027) of the K.U.Leuven, the Belgian Fund for Scientific Research - Flanders (G.0234.04 and G.0588.09), the Interuniversity Attraction Poles Programme - Belgian Science Policy (IUAP P5/04), the Flemish Regional Ministry of Education (Belgium) (GOA 2000/11), and the European Commission (STREP-2002-016276, IST-2004-027017, and IST-2007-217077).

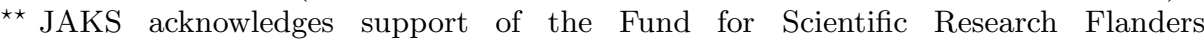
(G.0588.09).
} 
Brain computer interfaces are either invasive (intra-cranial) or noninvasive. The first ones have electrodes implanted usually into the premotor- or motor frontal areas or into the parietal cortex (see review in [2]), whereas the noninvasive ones mostly employ electroencephalograms (EEGs) recorded from the subject's scalp. The noninvasive methods can be further subdivided into three groups. The first group explores visually evoked potentials (VEPs) and they can be traced back to the 70s, when Jacques Vidal constructed the first BCI [3]. The second group of noninvasive BCIs rely on the detection of imaginary movements of the right or the left hand [4]. The third noninvasive group are the BCIs that rely on the 'oddbal' evoked potential in the parietal cortex, and is the topic of this article.

An event-related potential (ERP) is a stereotyped electrophysiological response to an internal or external stimulus [5. One of the most known and explored ERPs is the P300. It can be detected while the subject is classifying two types of events with one of the events occurring much less frequently than the other (rare event). The rare events elicit ERPs consisting of an enhanced positive-going signal component with a latency of about $300 \mathrm{~ms}$ [6].

In order to detect the ERP in the signal, one trial is usually not enough and several trials must be averaged. The averaging is necessary because the recorded signal is a superposition of all ongoing brain activities as well as noise. By averaging the recordings, the activites that are time-locked to a known event (e.g., onset of attended stimulus) are extracted as ERPs, whereas those that are not related to the stimulus presentation are averaged out. The stronger the ERP signal, the fewer trials are needed, and vice versa.

A number of off-line studies have been reported that improve the classification rate of the P300 speller [7, 8, 9], but not much work has been done on on-line classification. To the best of our knowledge, the best on-line classification rate for mind-typers is reported in [10]. For a decent review of BCIs, which is out of the scope of this study, see [11].

The BCI system descibed in this article is an elaboration of the P300-based BCI but with emphasis on a simple design for a power-efficient on-chip implementation.

\section{Methods}

\subsection{Acquisition Hardware}

The EEG recordings were performed using a prototype of an ultra low-power 8-channel wireless EEG system (see Fig. 1). This system was developed by IMEC partner and is built around their ultra-low power 8-channel EEG amplifier chip [12]. The EEG signals are $\mu \mathrm{V}$-range low-frequency signals that are correlated with a large amount of common-mode interference. This requires the use of a high performance amplifier with low-noise and high common-mode rejection ratio (CMRR). IMEC's proprietary 8-channel EEG ASIC consumes only $300 \mu \mathrm{W}$ from a single $2.7-3.3 \mathrm{~V}$ supply. Each channel of the ASIC consists of an AC coupled chopped instrumentation amplifier, a chopping spike filter and 

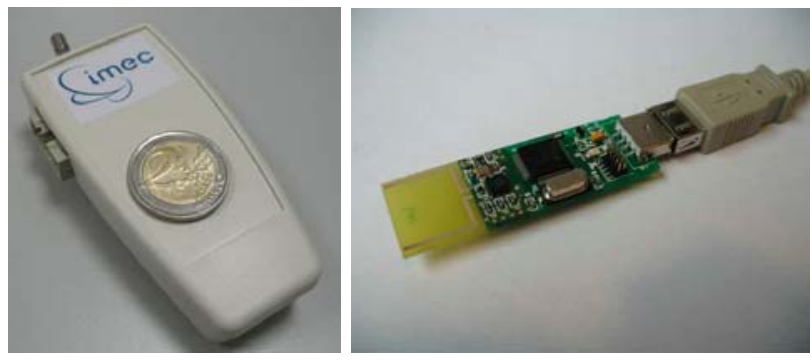

Fig. 1. Wireless 8 channel EEG system: amplifier and transmitter (left) and USB stick receiver, plugged into the extension cable (right)

a variable gain stage, and achieves $80 \mathrm{nV} / \mathrm{Hz}^{\frac{1}{2}}$ input referred noise density and $130 \mathrm{~dB}$ CMRR at $50 \mathrm{~Hz}$, while consuming $8.5 \mu \mathrm{A}$. The gain of the amplifier is digitally programmable between 2000 and 9000 and the higher cut-off bandwidth is digitally programmable between $52 \mathrm{~Hz}$ and $274 \mathrm{~Hz}$. The input impedance exceeds $100 \mathrm{M} \Omega$.

The wireless system uses a low-power microcontroller (Texas Instruments MSP430) combined with a low-power $2.4 \mathrm{GHz}$ radio (Nordic nRF2401). In operational mode, the EEG signal is sampled at $1000 \mathrm{~Hz}$ with 12 bit resolution. These samples are collected in packets and transmitted in bursts at a data rate of $1 \mathrm{Mb} / \mathrm{s}$ to the receiver, which is connected through a USB interface to the PC. The average total power consumption of the system in operational mode is $18 \mathrm{~mW}(6 \mathrm{~mA}$ at $3 \mathrm{~V})$. This implies it can be operated for more than one week continuously on two AAA batteries before the battery voltage drops below specification. At start-up, the system's parameters (such as gain and bandwidth settings) can be changed wirelessly.

The system also includes an electrode impedance measurement circuit that can measure the electrode impedances in the range of $1 \mathrm{k} \Omega$ to $6 \mathrm{M} \Omega$. The system is designed for use with $\mathrm{Ag} / \mathrm{AgCl}$ electrodes. For these experiments we have used a braincap with large filling holes and sockets for ring electrodes.

\subsection{Acquisition Procedure}

Recordings were collected from eight electrodes in the parietal and occipital areas, namely in positions $\mathrm{Cz}, \mathrm{CPz}, \mathrm{P} 1, \mathrm{Pz}, \mathrm{P} 2, \mathrm{PO}, \mathrm{POz}, \mathrm{PO} 4$, according to the international 10-20 system. The reference electrode and ground were linked to the left and right mastoids.

Each experiment started with a pause (approximately 90 second) needed for the stabilization of the EEG acquisition device. During this period, the EEG device transmits data but it is not recorded. The data for each symbol presentation was recorded in one session. As the duration of the session was known a-priori, as well as the data transfer rate, it was easy to estimate the amount of data transmitted during a session. We used this estimate, increased by a $10 \%$ margin, as the size of the serial port buffer. To make sure that the entire recording session 
for one symbol fits completely into the buffer, we cleared the buffer just before recording. This trick allowed us to avoid broken/lost data frames, which usually occur due to a buffer overflow. Unfortunately, sometimes data frames are still lost because of a bad radio signal. In such cases, we used the frame counter to reconstruct the lost frames, using a simple linear interpolation.

The overhead for one-symbol-session data reading from the buffer and the EEG signal reconstruction from the raw data, appeared to be negligible in Matlab, thus the latter was chosen as the main development environment.

\subsection{Data-Stimuli Synchronization}

Unlike a conventional EEG systems, the system we used does not have any external synchronization inputs. We tried to use one of the channels for this purpose (connecting it to a photo-sensor attached to the screen), but this scheme was not stable enough for long recording times. Finally, we came up with an "internal" synchronization scheme based on high-precision (up to hectananosecond) timing 1 .

For the synchronization, we saved the exact time stamps of the start and end points of the recording session, as well as the time stamps of stimulus onsets and offsets. Due to the fact that the reconstructed EEG signal has a constant sampling rate, it is possible to find very precise correspondences between time stamps and data samples. We used this correspondence mapping for partitioning the EEG signal into signal tracks, for further processing.

\subsection{Experiment Design}

Four healthy male subjects (aged 23-36 with average age of 31, three righthanded and one lefthanded) participated in the experiments. Each experiment was composed of one training- and several testing stages.

We used the same visual stimulus paradigm as in the first P300-based speller, introduced by Farwell and Donchin in [13]: a matrix of $6 \times 6$ symbols. The only (minor) difference was in the type of symbols used, which in our case was a set of 26 latin characters, eight digits and two special symbols '_' (used instead of space) and ' $\boldsymbol{~ ' ~ ( u s e d ~ a s ~ a n ~ e n d ~ o f ~ i n p u t ~ i n d i c a t o r ) . ~}$

During the training and testing stages, columns and rows of the matrix were intensified in a random manner. The intensification duration was $100 \mathrm{~ms}$, followed by a $100 \mathrm{~ms}$ of no intensification. Each column and each row flashed only once during one trial, so each trial consisted of 12 stimulus presentations ( 6 rows and 6 columns).

As it was mentioned in introduction, one trial is not enough for robust ERP detection, hence, we adopted the common practise of averaging the recordings over several trials before performing the classification of the (averaged) recordings.

${ }^{1}$ TSCtime high-precision time library by Keith Wansbrough http://www. lochan.org/2005/keith-cl/useful/win32time.html 
During the training stage, all 36 symbols from the typing matrix were presented to the subject. Each symbol had 10 trials of intensification for each row/column (10-fold averaging). The subject was asked to count the number of intensifications of the attended symbol. The counting was used only for keeping the subject's attention to the symbol. The recorded data were filtered in the $0.5-15 \mathrm{~Hz}$ frequency band with a fourth order zero-phase digital Butterworth filter, and cut into signal tracks. Each of these tracks consisted of 1000 ms of recording, starting from stimulus onset. Note that subsequent tracks overlap in time, since the time between two consequent stimuli onsets is $200 \mathrm{~ms}$. Then, each of these tracks was downsampled to 30 tabs and assigned to one of two possible groups: target and nontarget (according to the stimuli, which they were locked to).

After training the classifier, each subject performed several test sessions and where he was asked to mind-type a few words (about 30-50 symbols), the performance of which was used for estimating the classification accuracy. The number of trials $k$ that was used for averaging varied from 1 to 10 . For each value of $k$ the experiment was repeated and the classification accuracy was (re)measured. This experiment design differs from the one proposed in [10, where recordings and on-line classification were done only for $k=10$ and the evaluation of the method for the cases $k=1, \ldots, 9$ was done off-line using the same data. Our design is more time consuming, but it provided us with more independent data for analisys and evaluation, which is important in experiments with limited number of subjects.

\subsection{Classification}

In the proposed system the training stage of the classifier differs from its testing stage not only by the classification step, but also by the way of grouping the signal tracks. During the training, the system "knows" exactly which one of 36 possible symbols is attended by the subject at any moment of time. Based on this information, the collected signal tracks can be grouped into only two categories: target (attended) and non-target (not attended). However, during testing, the system does not know which symbol is attended by the subject, and the only meaningful way of grouping is by stimulus type (which in the proposed paradigm can be one of 12 types: 6 rows and 6 columns). So, during the testing stage, for each trial, we had 12 tracks (from all 12 types) of 1000 ms EEG data recorded from each electrode. The averaged (along trials) EEG response for each electrode was determined for each group. Then all 12 averaged tracks were sequentially fed to the feature extractor (see section 2.6 ), which extracted a scalar feature $y_{i}$ for each track $i$. In order to decide which symbol was attended, the classifier selected the best "row candidate" and the best "column candidate" among features $\left(y_{1}, \ldots, y_{12}\right)$ of all tracks, thus the row index $i_{r}$ and the column index $i_{c}$ of the classified symbol were calculated as:

$$
i_{r}=\underset{i=1, \ldots, 6}{\arg \max }\left\{y_{i}\right\}, \text { and } i_{c}=\underset{i=7, \ldots, 12}{\arg \max }\left\{y_{i}\right\}-6
$$


The symbol on the intersection of the $i_{r}$-th row and $i_{c}$-th column in the matrix, was then taken as the result of the classification and presented, as a feedback, to the subject.

\subsection{Feature Extraction}

In order to classify averaged and subsampled EEG recordings into target and nontarget classes we used the one-dimensional version of a linear feature extraction (FE) approach proposed by Leiva-Murillo and Artés-Rodríguez in [14]. As all linear FE methods, this method consider as features projections of the centered input vectors $\mathcal{X}=\left\{\mathbf{x}_{i}: \mathbf{x}_{i} \in \mathbb{R}^{D}\right\}$ onto appropriate $d$-dimensional subspace $(d<D)$, and the task is to find this subspace. The method searhes for the "optimal" subspace maximizing (an estimate of) mutual information between the set of projections $\mathcal{Y}=\left\{\mathbf{W}^{T} \mathbf{x}_{i}\right\}$ and the set of corresponded labels $\mathcal{C}=\left\{\mathrm{c}_{i}\right\}$. In the proposed system the class labels set consists of only two classes, thus we set $\mathcal{C}=\{-1,+1\}$. The dimensionality $d$ of the desired subspace was set to 1 , because it appeared to be enough to achieve a robust separation of the two classes, but compared to higher dimensionalities (we also tried $d=2,3$ ), it is computationally much cheaper. In the case of $d=1$ the subspace is represented

only by one vector $\mathbf{w} \in \mathbb{R}^{D}$ and projections are scalars $y_{i}=\mathbf{w}^{T} \mathbf{x}_{i} \in \mathbb{R}^{1}$. According [14, the mutual information between the set of projections $\mathcal{Y}$ and the set of corresponded labels $\mathcal{C}$ can be estimated as:

$$
I(\mathcal{Y}, \mathcal{C})=\sum_{p=1}^{N_{c}} p\left(\mathrm{c}_{p}\right)\left(J\left(\mathcal{Y} \mid \mathrm{c}_{p}\right)-\log \sigma\left(\mathcal{Y} \mid \mathrm{c}_{p}\right)\right)-J(\mathcal{Y}),
$$

with $N_{c}=2$ the number of classes, $\mathcal{Y} \mid c_{p}$ the projection of the $p$-th class' data points onto the direction $\mathbf{w}, \sigma(\cdot)$ the standard deviation and $J(\cdot)$ the negentropy estimated using Hyvärinen's robust estimator [15].

The input vector $\mathbf{x}$ is constructed as a concatenation of the subsampled and filtered EEG data: $\mathbf{x}=\left(x_{11}, x_{12}, \ldots, x_{1 K}, x_{21}, x_{22}, \ldots, x_{2 K}, \ldots, x_{N 1}, \ldots, x_{N K}\right)^{T}$, where $K$ is number of subsamples, and $N$ is number of channels. In our experiments we used $K=30, N=8$ and $D=K N=240$.

We also tried FE method proposed by Torkkola in [16, which in the onedimensional case is almost equivalent to the considered one, but it is slightly more computationally expensive. In multi-dimensional cases, the method by LeivaMurillo's outperforms Torkkola's method quantatively (in terms of the mutual information between classes and projections), as well as computationally (in terms of the number of floating point operations) as was shown by us in an earlier study [17.

\section{Results and Discussion}

The performance of each subject in mind-typing with our system is displayed in Fig. 2, where the percentage of correctly-typed symbols is plotted versus the 


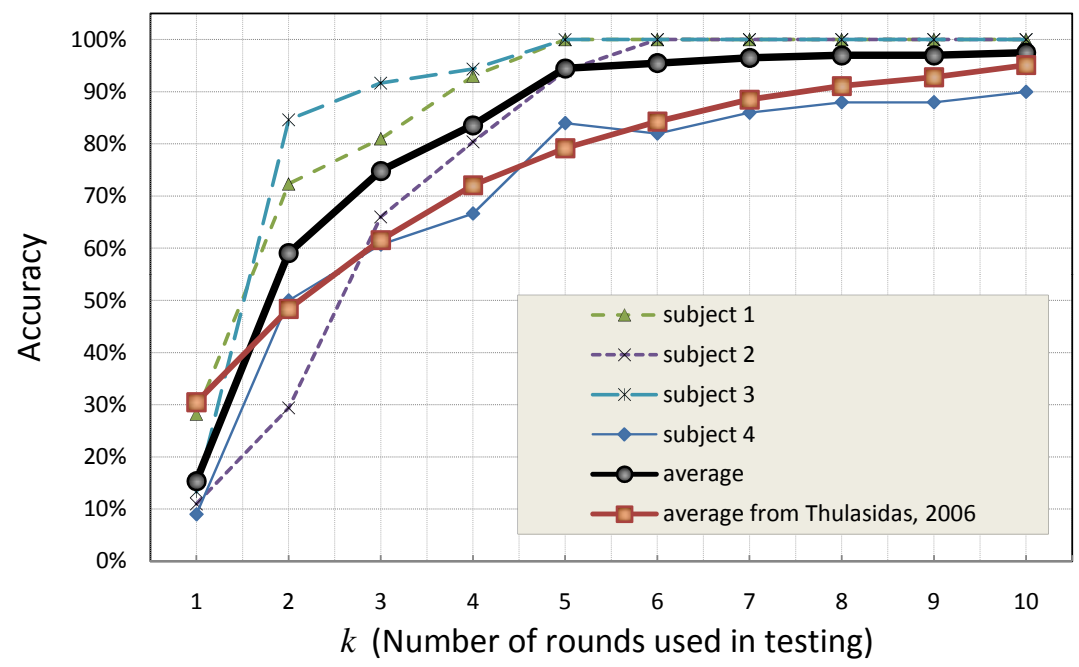

Fig. 2. Accuracy of classification for different subjects as a function of the number of trials used in testing. Averaged result and result from [10] are also plotted.

number of trials $k$ used for averaging. The average performance of all subjects, as well as the average performance of the best mind-typing system described in the literature 10, are also plotted. It should be mentioned that the mind-typing system of Thulasidas and co-workers is based on a support-vector machine (SVM) classifier with a Gaussian kernel, which is usually trained using a grid-search procedure for optimal parameter selection. The training of the SVM classifier with nonlinear kernel takes substantially more time than the training of the FE-based linear classifier used in our system.

Another consideration is that the on-chip implementation of the SVM classifier is more complex than our solution, due to the presence of nonlinearity in the kernel-based function.

As it is clear from Fig. 2, the performance strongly depends on the subject. However, we hasten to add that in order to draw any statistically-grounded conclusions from only four subjects, many more experiments need to be performed.

\section{Conclusions}

The brain-computer interface (BCI) presented in this article allows the subject to type text by detecting P300 potentials in EEG signals. The proposed BCI consists of a EEG system and a classifier which is based on linear feature extraction. The simplicity of the proposed system supports an efficient on-chip implementation (e.g., on an ASIC).

The results of this study shows that, in the field of BCIs based on event-related potentials, even simple solutions can successfully compete with the state-of-theart systems. 


\section{References}

[1] Editorial Comment: Is this the bionic man? Nature 442(7099), 109 (July 2006)

[2] Pesaran, B., Musallam, S., Andersen, R.: Cognitive neural prosthetics. Current Biology 16(3), 77-80 (2006)

[3] Vidal, J.: Toward direct brain-computer communication. Annual review of Biophysics and Bioengineering 2(1), 157-180 (1973)

[4] Birbaumer, N., Kubler, A., Ghanayim, N., Hinterberger, T., Perelmouter, J., Kaiser, J., Iversen, I., Kotchoubey, B., Neumann, N., Flor, H.: The thought translation device (TTD) for completely paralyzedpatients. IEEE Transactions on Rehabilitation Engineering 8(2), 190-193 (2000)

[5] Luck, S.: An introduction to the event-related potential technique. MIT Press, Cambridge (2005)

[6] Pritchard, W.: Psychophysiology of P300. Psychological Bulletin 89(3), 506 (1981)

[7] Kaper, M., Meinicke, P., Grossekathoefer, U., Lingner, T., Ritter, H.: BCI Competition 2003 - Data set IIb: support vector machines for the P300 speller paradigme. IEEE Transactions on Biomedical Engineering 51(6), 1073-1076 (2004)

[8] Serby, H., Yom-Tov, E., Inbar, G.: An improved P300-based brain-computer interface. IEEE Transactions on Neural Systems and Rehabilitation Engineering 13(1), 89-98 (2005)

[9] Xu, N., Gao, X., Hong, B., Miao, X., Gao, S., Yang, F.: BCI Competition 2003 - Data set IIb: enhancing P300 wave detection using ICA-based subspace projections for BCI applications. IEEE Transactions on Biomedical Engineering 51(2), 1067-1072 (2004)

[10] Thulasidas, M., Guan, C., Wu, J.: Robust classification of EEG signal for braincomputer interface. IEEE Transactions on Neural Systems and Rehabilitation Engineering 14(1), 24-29 (2006)

[11] Dornhege, G., Millán, J., Hinterberger, T., McFarland, D., Sejnowski, T., Muller, K.: Toward brain-computer interfacing. The MIT Press, Cambridge (2007)

[12] Yazicioglu, R., Merken, P., Puers, R., Van Hoof, C.: Low-power low-noise 8channel EEG front-end ASIC for ambulatory acquisition systems. In: Proceedings of the 32nd European Solid-State Circuits Conference. ESSCIRC 2006, pp. 247-250 (2006)

[13] Farwell, L., Donchin, E.: Talking off the top of your head: toward a mental prosthesis utilizing event-related brain potentials. Electroencephalography and clinical Neurophysiology 70(6), 510-523 (1988)

[14] Leiva-Murillo, J., Artes-Rodriguez, A.: Maximization of mutual information for supervised linear feature extraction. IEEE Transactions on Neural Networks 18(5), 1433-1441 (2007)

[15] Hyvärinen, A.: New approximations of differential entropy for independent component analysis and projection pursuit. In: Proceedings of the 1997 conference on Advances in neural information processing systems 10 table of contents, pp. 273-279. MIT Press, Cambridge (1998)

[16] Torkkola, K., Campbell, W.: Mutual information in learning feature transformations. In: Proceedings of the 17th International Conference on Machine Learning (2000)

[17] Chumerin, N., Van Hulle, M.: Comparison of Two Feature Extraction Methods Based on Maximization of Mutual Information. In: Proceedings of the 2006 16th IEEE Signal Processing Society Workshop on Machine Learning for Signal Processing, pp. 343-348 (2006) 